Studia UBB 政igitalia, Volume 62 (LXII) 2017, June, Issue 1, 89-99

Published Online: 2017-06-30

DOI:10.24193/subbdigitalia.2017.1.07

\title{
The importance of data visualization: teaching art history through timelines
}

\author{
Voica Pușcașiu \\ DigiHUBB, Babeș-Bolyai University, Cluj-Napoca, Romania, \\ E-mail: voicapuscasiu@gmail.com
}

\begin{abstract}
This research wishes to consider the venerable, but relatively marginal use of timelines as means of visualizing coexisting historical events and figures with special emphasis on Art History, a domain where it loses many of its inherent faults and manages to functional. The use of digital tools in humanities research is on the rise, yeilding interesting results as the timelines are now able to take on new forms. The benefits are thus increased and this chronological representation complements the traditional and established method, especially when it comes to teaching a subject that contains such ample amounts of data.
\end{abstract}

Keywords: timelines; data visualization; teaching; art history.

\section{Brief Introduction to Timeline Charts}

Without even bearing in mind the fact that we are leaving in an increasingly visual world, it has always been clear that visualization is one human beings' most basic tool in understanding the surrounding environment (Brisson 257). It is no coincidence that graphic schemes have been used to translate some of the most complex ideas, as they help in creating a much clearer view on the entire matter. Of course nowadays, this translation has been taken to new heights if we are to consider infographics as the fastest, most efficient way to deliver large amounts of data in the Information Age that has changed the way we think and communicate (Lankow, Ritchie and Crooks), an age of immediate gratification, distributive attention, and much less patience for text-heavy material. So if we are to accept that graphic visualization is among the most useful means in the better understanding of information (Admiraal 53) it should be no surprise that this is also valid for the field of History which deals with such large amounts of data. 
The visual method of presenting historical events through timeline charts came to be known in the 18th century as "chronography" from its combination of chronology and graphics (Admiraal 53), and it has largely stood the test of time, although it is surprisingly rarely used as a veritable teaching tool. Stemming from the genealogy and chronology charts of early 15th an 16th century, the most primitive shape they took was that of a table which contained the year on one column and the events on the second one, but from this, the timeline charts evolved in a multitude of styles, from the simple to the highly eccentric. One thing they do all have in common is the desire and intention to offer a global view of history of mankind as a whole. This is a seemingly impossible task, but it is made slightly easier through a graphic medium as a holistic view on such a large scale could not possibly be grasped otherwise. This desire to counteract the national discourses and the display of events grouped on geographical areas or certain time spans is beneficial for the realization that events and figures rarely stand on their own, but are interconnected in intricate ways.

The fact that complexity is often confusing is no less true for timeline charts, so it is obvious that most overly-zealous models are impractical due to the desire to include as much information as possible. This goes against the very idea of schematization and is one of the reasons some failed to get their point across, along with the biased that is sometimes present within them as they tend to give much more information about a specific era while ignoring others. The model that remained the most valuable due to its objectivity was given by Joseph Priestly in his New Chart of History (1769), which he dedicated to Benjamin Franklin (Jacobs 129), where he introduces a consistency of scale in his spatial representation of time that was not present before. The unvarying intervals that divide the timeline became the norm, as did the horizontal bands that indicate the lifespan of each person included in the chart, running from left to right in the direction of reading and it is this method that can be seen in the most basic linear chronologies that are present at the beginning of most history textbooks.

Chronology onto itself is one of the very first lessons introduced when learning about history (Păun 81), as placing an event in time is fundamental to its understanding, but this chronological approach underlined through the use of a simple timeline in not without its faults, mainly because it underlines a certain evolutionary aspect in history which is dangerous as it greatly diminishes the importance of later eras as compared to more recent ones. Perhaps even without setting out to do so, the focus on chronology creates the illusion of time passing from primitive societies all the way to the height of technology of the contemporary age and because of its schematic character it leaves no place for a more nuanced discussion. A second issue that might arise is the forceful implementation of a cause-and-effect principle (Stinespring and Steele), which although valid and of great importance for the study of history is not necessarily true in all situations, just because an event preceded another it does not automatically means 
it also caused it so this possible reading needs to be taken into account whenever creating or analyzing a timeline chart. Thus the most useful graphics are not the ones that simply visually represent a succession of facts or figures, but the ones that are multi-layered, including multiple bands representing different geographical zones and if possible they should strive for interdisciplinarity in order to offer an ampler context to events and manifestations.

\section{Timelines in Art History}

Concerning the field of Art History, asides the "history-as-evolution" issue, many caveats of the timelines are lost, especially when comparing them to the real benefits this type of visualization can bring when dealing with very specific causeand-effect scenarios that are inevitably present and important. But once again their presence is not as widespread as one would expect, despite being used by some of the most influential authors, mostly in the works that were designed for educational and popularization. Although not textbooks per se, the books containing timelines were used for this purpose and include some of the best-selling Art History volumes of all times such as H.W. Janson's History of Art (Janson) and Ernst Gombrich's The Story of Art (Gombrich) both of which have kept and refined the timelines section throughout their many editions. Besides offering a more global picture of the artistic manifestation, these graphic representations offer at a glance the artists and their contemporaries, which as Priestley said, gives "a peculiar kind of pleasure" when one imagines the possible conversations (Priestley qtd. in Jacobs 129). This instant emotional connection is incidentally another one of the visualization's benefits, which would be hard to achieve otherwise.

A problem that is specific for Art History, but finds a solution through the use of the timeline charts is the different speeds at which a certain style reached different geographical areas. The traditional and well established manner of writing about Art History is rarely more than a succession of styles, and even though they are sometimes differentiated by location this can prove both hard to understand and easy to forget. The most obvious case is that of the Renaissance whose ideals and manifestations are considered to have appeared with some delay in Northern Europe and kept a rather Gothic appearance for even longer than that, Janson himself created two separate timelines regarding Italy and the Northern Countries in order better to underline this issue (Bork 182). This however seems unsuitable, because if they were to be presented on the time scale, an even better appreciation of this delay could be achieved, as it would give the viewers the occasion to realize that Jan van Eyck, a pioneer of the portrait, was long dead before Leonardo da Vinci was even born despite the fact that in the usual Art History discourse van Eyck follows da Vinci. A different means of 
reaching conclusions that would be hard to come by through traditional methods is if one would choose to point out on the timeline specific artworks and analyze their similarities and differences. A good example in this case would be Raphael's Crucifixion (1503) versus Matthias Grünewald's Crucifixion (1502), created just one year apart they have the same theme and a strikingly similar composition, and yet there is absolutely nothing of Raphael's serene color scheme and blissful figures in Grünewald's gruesome depiction of torment.

A different side of this issue is the customary division of the Baroque style based upon the different countries it reached, usually starting with Italy, continuing with Spain, The Netherlands, and so on. This flow of the discourse that seems to end each chapter before starting a new one is disruptive simply because it tends to overlook the concomitance of artists from different geographical locations and their possible interactions and influences on each other. What's more, the stylistic division in itself is problematic because it mostly overlooks the artists or works that are on the fringes of a certain current, or more frequently they get labeled into either one of the styles while ignoring the characteristics of the other. Through the use of timelines, these works could more easily show the way styles flowed and not abruptly ended in order to give way to the next one. This approach, based on concomitance and correspondence is notably used by Gombrich but is surprisingly rare afterwards, except in Julian Bell's book (Bell), and almost never in textbooks. Even rarer are the visual schemes that depart from the chronological timelines in order to show the influences one current has on others, or that others have had on it, but such examples do exist, an original and entertaining one being that of Alfred $\mathrm{H}$. Barr Jr. concerning Cubism and modern abstract art (Jacobs 133). This goes to show that visual aids in Art History should not always be contained to Priestley's model in order to be effective, but that there are many more imaginative ways through which a valuable point could be made.

Besides Barr's suggestion of graphic representation that raises awareness on the many obscure artistic subdivisions that hardly ever get mentioned, even when it comes to timelines there are a number of different types that could be thought up in order to serve as many different purposes, depending on the discourse it is associated to, be it explicit or implicit. One of the ways in which timelines could be constructed implies following the artists' lives and pinpointing personal events that are thought to have influenced their work. This style could work if one starts with the presumption that personal events shape an artist's work, as some art historians do, and they would be accompanied by relevant examples from their oeuvre that prove this point. The events on the timeline could range from the death of a loved one, to happier events and from the artist's educational background to his moving to a different location and coming into contact with a different culture. This type of timelines would be instrumental in establishing the course of an artist's work and view the stylistic 
and thematic changes it has gone through. As it is highly unlikely that a single event could provoke an instant change in an artist's style, these should only be seen as triggers towards change, while the timeline could also lead to a better appreciation of the lifelong artistic exploration of a certain theme of motif instead of just breaking up the entire volume of one's work into definite periods. Of course following the stylistic transformations of an artist's work does not necessarily have to be connected to his personal life or any other external events, a simpler version of this type of timeline could include just the works set up in a chronological manner, from which one could presumably get a better grasp on the nature of these transformations.

A different aspect of timelines could be used by those interested in establishing the iconographical changes (Szabo 159), through the ages by considering specific works that follow the same pattern, either through theme, style, compositions or the symbols they use. In this sense thematic timelines would pinpoint the most relevant artworks in order to offer a quick view of the entire history of one genre, while at the same time creating a clear connection between the artists who influenced each other. The possibilities for these timelines are endless and they could be as broad as the representations of a fruit bowl from Ancient Egypt to Matisse, or as narrow as the depictions of Napoleon in various stances. Even when tackling one genre in particular the examples can be broadened to all types of female nudes throughout history, regardless of the medium, or more focused on a precise characteristic such as the reclined nude. In this case a timeline could point out Giorgione's Sleeping Venus (1507), Titian's Venus of Urbino (1538), Velázquez's Rokeby Venus (1651), Ingres' Grand Oladissque (1814), Manet's Olympia (1863), Cézanne's A Modern Olympia (1873), and ending with the work of Guerilla Girls Do women have to be naked to get into the Met. Museum? (1989).

Much rarer, but much more useful are the timeline charts that while referring to Art History also choose to present things in a larger context, by offering a interdisciplinary view of a time section. Such examples can be found in Norbert Wolf's book on Romanticism (Wolf) that offers information on activities connected to art on a timeline that is placed at the bottom of each page and runs through the entire volume or David Gariff's endeavor (Gariff) of establishing artistic influences based solely on graphic representations of timelines. The utility of these charts is clearly seen if one is to consider the large effect the domains outside of art have on artist production throughout the ages all of which could be shown in a simple and efficient manner. Considering the fact that a great deal of art is not self-referential, but is inspired by various fields and events, it makes perfect sense to include these in order to gain a truly global view on Art History. By and large the most common influence comes from historical events, be they wars, inventions or relevant figures, all of which have prompted very specific manifestation in the visual arts, as well as in 
literature and music. The problem is that these connections are more or less direct and they are not always easy to be touched upon in a coherent manner, thus a visualization of selected and pertinent data is preferable (Szabo 129). If in the case of history the influence upon art is unilateral, as significant events have rarely been precipitated by a work of art, when it comes to literature and music, the influences are mutual and there are many examples of this practice, so in order to fulfill a truly global purpose, layers including these fields could also be added to the timeline.

Accepting Arnold Hauser's thesis on the fact that all art is fundamentally connected to the society in which it was created (Hauser 6), it becomes obvious that not enough is being done in order to underline this causality. The fact that (Art) History is perceived as a series of epochs and (art) historians are specialized in a narrow section (Bork 179), creates the false impression of a clarity of boundaries which is detrimental for those who are not yet well-versed in the intricacies of these fields. It is for this reason that visualization could become a practical teaching instrument, that while covering an ample set of information, is not as dense and demanding, but rather "user-friendly". Interdisciplinary timelines are even more important because they are able to offer a cross-section of any given time period and paint a much more comprehensible picture of it. For example considering the time surrounding the French Revolution, a single visualization could mark the Storming of the Bastille (1789) but also what that has meant to for the arts: La Marseillaise (1792), Delacroix's Liberty Leading the People (1830), and Hugo's Les Miserables (1862), all four of which would have traditionally been discussed at different times, during the study of different subjects. What is even better is the fact that timelines are a universally applicable method that could be used for either for specific welldefined situations, or for a more general aspect, thus creating a sense of structure, also by containing less text but more detail they are superior to regular charts that are most often found in history textbooks (Stinespring and Steele 9).

Each of the methodologies for creating a timeline, briefly discussed above can have as a result a tool that could be used for either education or even just for broadening the general interest towards this field, it is however important to accept that as a schematic representation some things have to be left out (Admiraal 56), and mere existence in not a criteria for inclusion. As clutter is one of the most counterproductive aspects of a visual representation and history textbooks are notoriously crammed with too much data (Felezeu 107), it would be the timeline's task to right this wrong and not concentrate on isolated details. One of the most important didactic principles that is delivered through timeline charts is that of comparison (Felezeu 183), this refers to two different procedures, both of which could be achieved. First there is precisely the one discussed earlier in this paper concerning similar events of manifestations that happened at very different historical times and 
the second one refers to simultaneous comparison, involving events and phenomena that happened in the same time span but under very different circumstances. A prime example for this could be the existence of a Baroque style in both Catholic and Protestant countries. Finally not only do timelines serve an educational purpose, but they are also visually attractive, which is of utmost importance especially nowadays when society is surrounded by glossy, colorful prints and images that captivate the eye and the mind.

\section{Digital Art History}

Data visualization was a term initially used by statisticians (Francis and Fuller 301 ), but has since become a modern term for a graphic representation of a set of data. It can be used in reference to a "hand-made" graphic display, but through recent developments, it has also become associated with software-generated images and charts that provide insight into scientific data, though traditionally used for engineering, medicine, and geology, this approach has slowly started to appear in humanist fields as well as of late. Despite the boom in technology that greatly affected the way graphic information in being presented, some traditional mediums have still remained viable and timelines are notably one of them (Admiraal 53). The timeline chart appears to have successfully passed the test of time and the translation to a digital environment which means that all its best traits are still sought after, underlining undeniable the fascination for its elements, be it the glimpse into simultaneous events, their simple and logical chronology, their application of the cause-and-effect principle, or just the fact they are intuitive to use. Whatever the reason it is quite clear that they are very much in use today and the existence of software such as Timeline JS just proves there is a market for it and that people are more than willing to create their own charts, often for educational or presentation purposes.

In order for generated timelines to be considered efficient it still needs to follow the same characteristics as before, it should be clear and appropriate for communicating the information as its success is mainly determined by the speed at which it manages to do just that. Even though its main goal still resides in conveying a message or showcasing patterns hidden in the data (Ramirez Gaviria 479), when compared to older, hand-made timelines, it becomes clear that new technology brought along a more aesthetic dimension into all visualizations, and it is through this aspect that innovative ways of presentation could eventually be reached, instead of relying on the same model that dates from the 1700s (Jacobs 133). What is more important is that even though it is still true that not all data could (or should) be contemplated at once, computer visualizations have the added bonus of being dynamic and interactive, so through filters, panning, and zooming the viewer can choose what 
to see at a given time, according to his interest, while the rest of the data, though still present and available will be hidden from view. One can imagine in the case of Art History a timeline of an artists' life containing each and every one of his works in chronological order, but while a complete visual would be overwhelming, the viewer could choose to view only the works from a specific year, for example. This dynamic data visualization is considered to be one of the only genuinely new cultural forms enabled by computing (Manovich 3 ), the possibilities it offers are virtually endless and contemporary timelines have started to make use of these new prospects.

Without going into great detail, two timeline-based projects should be mention and even though many others exist, they are all only starting to scratch the surface of what could be done through this medium. The first and probably one of the most famous is the Heilbrunn Timeline of Art History hosted by the website of the Metropolitan Museum in New York. It was launched in 2000, spanning from 8000BCE until the present day and contains over 6000 photographed works of art from the museum's vast collection (Van Kirk 290). It is also accompanied by an interactive map of the world so that location can be used as search criteria, and it compiles 300 separate timelines that are stacked or layered for comparison (Knapp 28), and it is enhanced with so-called Thematic Essays, providing an engaging way to do research or just browse the collection. Data is always being added as well as practical information about which pieces are on display and where, and which are currently in storage, so it fulfills a secondary role in helping plan a visit to the museum. Of course this extensive and impressive project could only be upheld through an equally impressive funding in this case provided by Heilbrunn Foundation, New Tamarind Foundation, and Zodiac Fund, to which such a renowned institution is likely to have access.

Other examples are on a much smaller scale, but no less interesting and one of them is Giorgia Lupi's Visualizing Painters' Lives (Lupi) done in collaboration with illustrator Michela Buttignol. The project is made out of 10 separate timelines, each belonging to a painter, and the idea behind it was to build a "visual anthology" of some of the most recognizable painters of the modern era by borrowing specific pictorial elements from each artist in order to tell their story. Although each one marks events with unique symbols, the timelines are all structurally uniform, so they also function as a whole, as a series. What makes them particularly interesting is that they don't only contain details about artistic production in recognized stylistic periods of a painter's life, but also pinpoint his education, training, awards, as well as connections and influences. There is at the same time a biographical aspect to them in the information about the artists' habits, health, travels, and romantic connections, in order to offer a view of the man as well as the professional, all in one elegant, whimsical, and informational panel. This kind of projects push the boundaries of both Art History and data visualization, striving for both clearer information and better 
design, thus software development could be combined with a comprehensive database, this should currently be priority that begs the collaboration of all institutions in order to digitize their precious collections.

In order to be able to speak of a proper Digital Art History, the developments in the field of Digital Humanities are a good start, but not nearly enough so Murtha Baca and Anne Helmreich have identified five steps, or phases, that need to be completed in order to go from digitizing to fully digital (Baca and Helmreich qtd. in Ross). The very first step is well on its way with many initiatives such as Artstor, the Google Art Project, and that of the Getty Research Institute, along with many other more focused ones like The William Blake Archive or Vincent van Gogh: The Letters, which not only make valuable resources digitally available but enhance the regular viewer experience by offering impeccable resolutions of the pictures that are now suitable for an whole range of analyses or even just for personal entertainment. The second step towards Digital Art History involved the creation of specific tools in order to provide the infrastructure for building a collection, accessible to the individual scholar, this way collaboration would be encouraged and students could easily become producers as well as consumers (Baca and Helmreich qtd. in Duggan). This step materialized with the appearance of software like Zotero and Omeka, both of which have been designed with scholars in mind in order to help with creating and sharing collections. Phase three supposedly involves the creation of new technologies for visualization, reconstruction, and virtual reality, which would mean that works could be understood in a very different manner that it was possible until now, and although there are projects in this direction, like Virtual Burnham Initiative, the process seems to have stalled as they most rely on the concept of curated collection. It is the fourth step however that would have a true implication for the future of this field as it would involve new means of scholarly publishing and peer review, which along with the fifth and final phase of using new models of scholarly research enabled by computational analyses, would really change the way in which Art History is experienced and thought while harnessing the full potential of computers for this field and its scholars.

The direction in which research is seems to be headed in our contemporary society could bring new and unexpected forms and scholars and educators should be able to adapt to this new state of things, especially when it comes to teaching generations that are "born digital" it is however obvious that the past still has worthy models that first need to be known and experienced in order to be reinterpreted and re-imagined through technology. Timelines have much to offer and it appears that they have not reached their full potential, so it is a direction worth following for the future of the Digital Humanities. 


\section{Works Cited}

Admiraal, Ashley. "The History Timeline Chart". Agora. Vol. 47. No. 1 (2012): 53-59. Bell, Julian. Oglinda lumii. București: Vellant, 2007.

Bork, Robert. "Pros and Cons of Stratigraphic Models in Art History" RES: Anthropology and Aesthetics. No. 40 (Autumn 2011): 177-187.

Brisson, Harriet E. "Visualization in Art and Science". Leonardo. Vol. 25. No.2/3. Visual Mathematics: Special Double Issue (1992): 257-262.

Duggan, Bob. "What would Digital Art History Look Like?". April 212017. <http://bigthink.com/Picture-This/what-would-digital-art-history-look-like>.

Felezeu, Călin. Didactica istoriei. Presa Universitară Clujeană: Cluj-Napoca, 2000.

Francis, Brian and Mark Fuller. "Visualization of Event Histories". Journal of the Royal Statistical Society, Series A (Statistics in Society). Vol. 159. No. 2 (1996): 301-308.

Gariff, David. Cei mai influenți pictori din lume și artiștii pe care i-au inspirat. Enciclopedia RAO: București, 2008.

Gombrich, Ernst. Istoria artei. București: Pro Editură și Tipografie, 2007.

Hauser, Arnold. The Philosophy of Art History. Cleveland and New York: Meridian Books, 1967.

Jacobs, Alan. "History as Wall Art". The New Atlantis, A Journal of Technology \& Society. (Fall 2010): 127-133.

Jansen, Leo, Hans Luijten, and Nienke Bakker (Eds.). Vincent van Gogh: The Letters. April 21 2017. <http://vangoghletters.org/vg/>.

Janson, H.W. and Anthony F. Janson. History of Art. Eight Edition. New York: HN Abrams, 1991.

Knapp, Jessica. "Clay Culture: Timeline of Art". Ceramics Monthly. June/July/August (2012): 28.

Lankow, Jason, Josh Ritchie and Ross Crooks. Infographics: The Power of Visual Storytelling. New York: John Wiley \& Sons, 2012.

Lev Manovich. "Data Visualization as New Abstraction and as Anti-Sublime". Small Tech. The Culture of Digital Tools. edited by Byron Hawk, David D. Rieder, and Ollie Oviedo. Minneapolis: University of Minnesota Press, 2008: 3-9.

Păun, Ștefan. Didactica istoriei. București: Corint, 2001.

Ramirez Gaviria, Andres. "When is Information Visualization Art? Determining the Critical Criteria”, Leonardo. Vol. 41. No. 5 (2008): 479-482.

Ross, Nancy. "Teaching 20th Century Art History with Gender and Data Visualization". The Journal of Interactive Technology and Pedagogy. Issue 4 (Fall 2003).

Stinespring, John A. and Brian D. Steele. "Teaching Art History: Getting Started". Art Education. Vol. 46. No. 2. Art History (March 1993): 7-13. 
Szabo, Victoria. "Transforming Art History Research with Database Analyses: Visualizing Art Markets". Art Documentation: Journal of the Art Libraries Society of North America. Vol. 31 (Fall 2012): 158-175.

Van Kirk, Shannon. "Heilbrunn Timeline of Art History". College and Research Libraries News. May (2012): 290-291.

Wolf, Norbet. Romanticism. Cologne: Taschen, 2007.

Giorgia Lupi. Visualizing Painters's Lives. April 20 2017. <http://giorgialupi.com/ work\#/visualizing-painters-lives/>.

Timeline JS. April 20 2017. <https://timeline.knightlab.com/>.

Art Project - Google. April 21 2017. <https://www.google.com/culturalinstitute/project/ art-project>.

Artstor. April 21 2017. <http://www.artstor.org/>.

Getty Research Institute, The. April 21 2017. <http://www.getty.edu/research/scholars/ digital_art_history/index.html>.

Metropolitan Museum of Art, The. April 21 2017. <www.metmuseum.org/toah/>.

Omeka. April 21 2017. <http://omeka.org/>.

Virtual Burnham Initiative. April 21 2017. <http://vbi.lakeforest.edu/>.

William Blake Archives, The. April 21 2017. <http://www.blakearchive.org/blake/>.

Zotero. April 21 2017. <https://www.zotero.org/>. 